\title{
Spectral Imaging of Individual Split-Ring Resonators
}

\author{
Guillaume Boudarham, ${ }^{1}$ Nils Feth, ${ }^{2}$ Viktor Myroshnychenko, ${ }^{3}$ Stefan Linden, ${ }^{2,4}$ Javier García de Abajo, ${ }^{3}$ \\ Martin Wegener, ${ }^{2,4,5}$ and Mathieu Kociak ${ }^{1, *}$ \\ ${ }^{1}$ Laboratoire de Physique des Solides CNRS/UMR8502, Bâtiment 510, Univ. Paris-Sud, Orsay, 91405, France \\ ${ }^{2}$ Institut für Angewandte Physik, Universität Karlsruhe, Wolfgang-Gaede-Strasse 1, 76131 Karlsruhe, Germany \\ ${ }^{3}$ Instituto de Óptica, CSIC, Serrano 121, 28006 Madrid, Spain \\ ${ }^{4}$ Institut für Nanotechnologie, Karlsruhe Institute of Technology, 76021 Karlsruhe, Germany \\ ${ }^{5}$ DFG-Center for Functional Nanostructures, Karlsruhe Institute of Technology, 76021 Karlsruhe, Germany \\ (Received 29 September 2010; revised manuscript received 17 November 2010; published 14 December 2010)
}

\begin{abstract}
We report on spectral imaging within individual silver split-ring resonators (SRRs) operating in the near infrared-visible range. We classified the optical eigenmodes from the measurement of their energies and nanometer scale spatial distributions. They are plasmonic standing waves that show great similarities with that of nanoantennas. We, however, evidenced marked differences in the near-field electric field lines' spatial distribution and the energies' dispersion. We also showed that the subwavelength defect's influence on the SRRs' eigenmodes spatial distribution is small.
\end{abstract}

DOI: 10.1103/PhysRevLett.105.255501

PACS numbers: 81.05.Xj, 73.20.Mf, 79.20.Uv

Metamaterials are artificial structures made up of the repetition of man-made elements, often called photonic atoms, in analogy to the real atoms of conventional materials. They exhibit a number of extraordinary properties that are not available in natural materials, including negative phase velocity of light [1]. They are currently finding useful applications in artificial magnetism, perfect lensing, and cloaking [2,3]. Like real atoms, photonic atoms are much smaller than the light wavelength at which they operate, but unlike real atoms, their physical properties are mainly governed by Maxwell's equations rather than Schrödinger's equation. Real atoms can be entirely described given the energy of their eigenstates and the spatial distribution of their associated wave functions. Experimental access to the variation of the wave functions at different energies, namely, performing spectral imaging, has been only recently achieved on real atoms [4,5]. This has given rise to useful insight on the material they are built in. Surprisingly, spectral imaging of photonic atoms has still not been achieved, despite having much larger size (typically more than $100 \mathrm{~nm}$ ). Indeed, until recently, subwavelength optical spectroscopy was still not sufficiently advanced, and the physical realization of such small structures is technologically challenging. In order to deal with this issue, we have considered the paradigmatic case of the split-ring resonator (SRR), basically a cut winding loop of metal.

Indeed, they exhibit an artificial negative permeability [6] which does not appear easily in ordinary materials, in a range spanning from microwave to visible that can be tuned by changing the dimensions of the SRR. Moreover, together with a negative permittivity, they allow a negative phase velocity [1] of light even at optical frequencies.

The need for a complete experimental characterization of these photonic atoms acting in the visible range is particularly important. In this range, the operating frequencies reach the plasmon frequency of the underlying metal and the inertia of electrons shows up so that the optical excitations now have dispersion relations markedly different from free light in vacuum and highly dependant on the aspect ratios of the nanostructure [7,8]. Also, the energy dissipation has to be taken into account. The simple picture borrowed from electrical engineering where the $U$ shape of the SRR can be approximated by a $3 / 4$ winding loop [9] totally misses the plasmonic nature of the photonic atoms' eigenmodes [10].

In this Letter, we present an experimental and simulation study of the near-field properties of the eigenmodes of individual silver split-ring resonators that operate from the near-infrared to visible range. This was achieved by performing spatially resolved electron energy loss spectroscopy (EELS) in a scanning transmission electron microscope $[8,11,12]$ on adapted $e$-beam-lithographed samples. We obtained the energies and spatial distribution of the optical eigenmodes of the SRRs. The eigenmodes could then be classified through their energies and symmetries, and have strong similarities with that of nanoantennas. However, thanks to the high spatial resolution obtained, we could clearly evidence a deviation of the SRRs eigenmodes near-field properties with respect to the nanoantenna ones and the effect of the coupling between legs of the SRR on the dispersion curves. Also, the effect of subwavelength defects could be directly probed and was shown to be small.

We adapted the $e$-beam lithographic process recently developed for fabricating photonic atoms in the visible range [13] to a substrate transparent to fast electrons and thus compatible with EELS (a suspended $30 \mathrm{~nm}$ thin $100 \times$ $100 \mu \mathrm{m}^{2} \mathrm{Si}_{3} \mathrm{~N}_{4}$ membrane window sitting on a holey silicon frame). Briefly, a thin PMMA resist is spin cast, 
and then exposed along a defined pattern to a $30 \mathrm{kV}$ electron beam. After developing the resist, a $20 \mathrm{~nm}$ thin silver layer is deposited by electron-beam evaporation and the resist layer is removed by a lift-off procedure. We note that EELS on $e$-beam-lithographed samples has not been reported before. Our method will open to the large collection of $e$-beam-lithographed samples of interest in optics the high spatial resolution characterization ability of the EELS techniques. We used a scanning transmission electron microscope (STEM-VG HB 501) at normal incidence operating at $100 \mathrm{keV}$ and equipped with an EELS spectrometer and a homemade CCD camera. The size of the probe is $1 \mathrm{~nm}$ and the energy resolution in the raw data is $0.4 \mathrm{eV}$. The experiments are performed as explained elsewhere [14]. Briefly, a set of spectra is acquired by scanning the electron beam with a constant spatial step close to $1 \mathrm{~nm}$ and taking $50 \mathrm{spectra/pixel.} \mathrm{Typical} \mathrm{ranges} \mathrm{are} 32 \times 32$ pixels and $1 \mathrm{~ms} /$ spectrum. The spectra are a posteriori spectrally aligned, summed, and deconvoluted from their point spread function [15]. This allows us both to increase the energy resolution to $0.3 \mathrm{eV}$ and to remove the background due to elastically scattered electrons. For each spectrum of the data set, each peak is automatically detected and subsequently fitted with a Gaussian profile [14]. EELS intensity maps are then generated. Finally, boundary element method (BEM) simulations are performed for the EELS intensity spectra and energy-filtered maps, as well as for near-field distributions of the modes in the rings, as described elsewhere [16-18].

We studied four SRRs of similar dimensions (width $\times$ height $\times$ thickness $\approx 145 \times 130 \times 20 \mathrm{~nm}^{3}$ ) [see Fig. 1(a)]. Figure 1(b) shows the spectra taken at different positions along the SRR profile of the sample labeled SRR1. The EELS peaks can be classified into three families. The first two of them are denoted $A\left(A_{1}, A_{2}\right)$ and $S\left(S_{1}, S_{2}\right)$ for reasons that will appear clear below. The third family is a bunch of high-energy modes spanning the $2.5-4 \mathrm{eV}$ range. We are interested in the optical region for which these structures find application as pillar stones of metamaterials designs, and therefore, we will not enter into a discussion of these high-energy modes, other than indicating that their surface or bulk origin is well established by comparison with calculations [see Fig. 1(c)]. It is clear from Fig. 1(b) that the peaks exhibit some specific spatial symmetries at least for $S$ and $A$ modes. In order to clarify this point, we plot in Fig. 2(a) the EELS intensity maps for $A$ and $S$ modes, which have clearly discernible behaviors. Following the U-shaped SRR profile, the four first resonances exhibit a decreasing distance between resolved spatial maxima as the energy increases. Our BEM simulations yield excellent agreement with experiments, especially for the spatial distribution of the modes. In particular, it shows that the rather counterintuitive lack of intensity for $A_{2}$ and $S_{2}$ modes at the tips of the $U$ shape are not an experimental artifact [see Figs. 2(a) and 2(b)]. Moreover, it should be noted that the EELS signal is concentrated on the outer part of the SRRs for some maxima [see Fig. 2(a)]; this behavior is very well reproduced in our numerical simulations [see Fig. 2(b)]. The maps of both $S$ and $A$ modes are symmetric with respect to the central axis of the SRR, but only the $S$ modes display a maximum along this axis. The EELS signal is closely related to the electromagnetic local density of states, polarized along the $z$ electron axis trajectory (perpendicular

\section{Experiment}

(a)

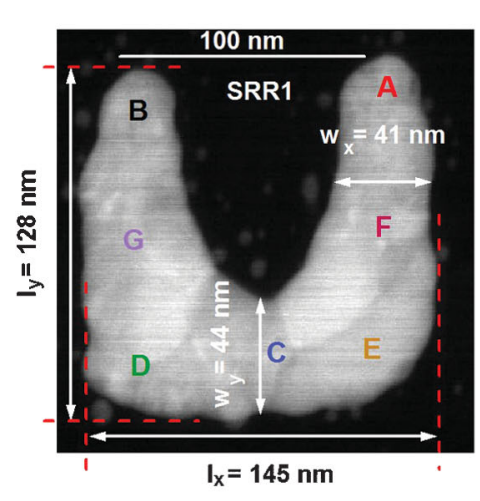

(b)

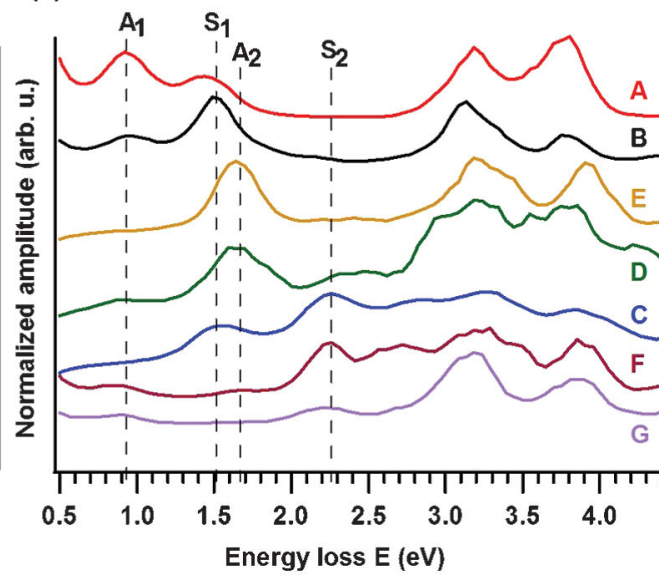

(c)
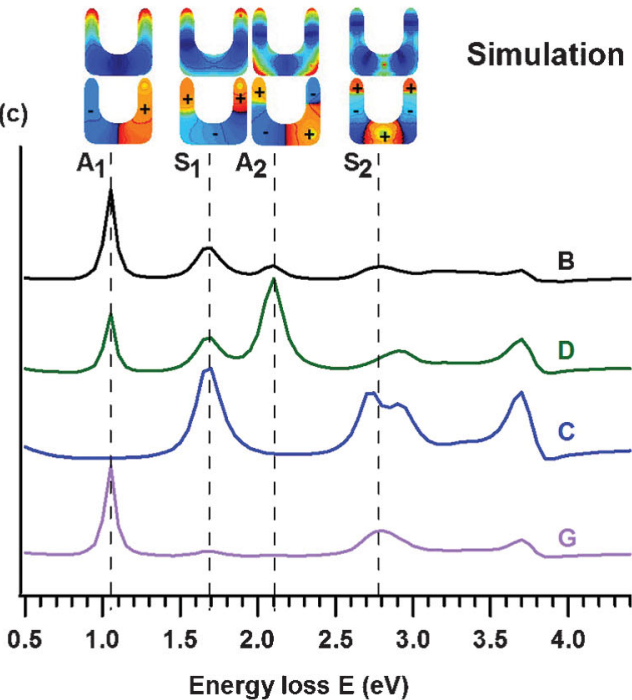

FIG. 1 (color). Resonance energies in a single split ring. (a) High angle annular dark field (HAADF) image of sample SRR1. (b) EELS spectra obtained from deconvoluted raw data taken at the positions A-G, as indicated in (a). (c) EELS spectra obtained from simulation for a symmetric SRR (width $\times$ height $\times$ thickness $\approx 145 \times 136 \times 20 \mathrm{~nm}^{3}$ ). Each spectrum has been normalized to its maximum amplitude. The spectra are vertically offset for clarity. The 2D-color plots above the spectra show the modulus (upper row) and the phase (lower row) of induced charges of plasmon modes for a SRR. 


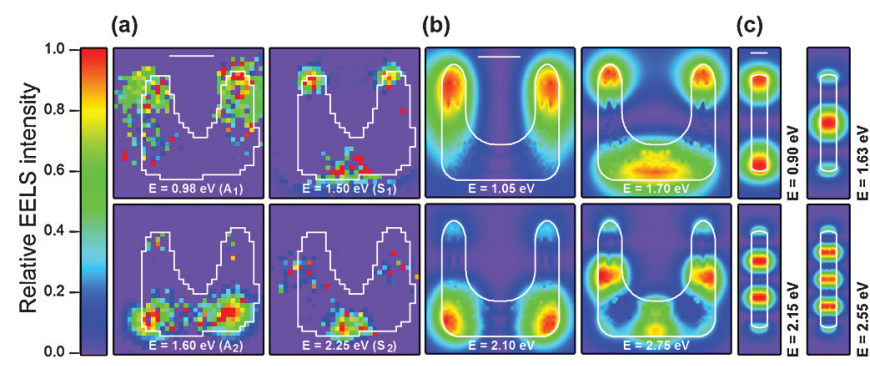

FIG. 2 (color). EELS intensity (amplitude $\times$ FWHM) maps of the resonances resolved for the sample SRR1. Experiments (a) are compared with BEM simulations for a SRR (b) and a nanoantenna $(\mathrm{c})$ (scale bar $=50 \mathrm{~nm}$ ). The latter does not incorporate the effect of the substrate. The ratio between maxima compared with $A_{1}$ is (ordered by increasing plasmons energy): experiment: $1|2| 3 \mid 4$; theory for SRR: $1|1| 9 \mid 1$; theory for nanoantenna: $1|1| 0.5 \mid 0.3$.

to the rings), i.e., the $z$ EMLDOS [19]. In other words, the displayed maps are roughly following the square of the electric field projected along the electron path, and is thus not sensitive to the phase of the eigenmodes. However, from the fact that the $A$ modes do not have maxima at the symmetry axis, in contrast to $S$ modes, we can infer that $S$ modes are symmetric in charge with respect to the symmetry axis of the SRR, whereas $A$ modes are antisymmetric. This is in agreement with both of our BEM simulations (see inset of Fig. 1(c)] and previous calculations [10] and experiments [20]. All of these resonances are still present for SRRs with slightly different sizes and shapes [see Fig. 3(a)].

The classification of the SRR modes can be done either based on a phenomenological model of alternative current distributions along the $U$ path [13], or equivalently, based on the near-field distribution induced by the charges and currents associated to the modes [10]. In both cases, the concept emerges that the various modes are different orders of a standing wave along the $U$ path of the SRR, explicitly described as surface plasmons in [10], and exhibiting a different symmetry with respect to the central axis of the $U$ shape. Noting that the EELS intensity is closely related to the zEMLDOS, the maps of Fig. 2 can be directly interpreted as reflecting plasmonic standing wave oscillations. We are now in a position to classify all modes according to the symmetry of these oscillations. $A_{1}$ is related to the so-called "magnetic mode" (or " $L C$ resonance" in electrical-engineering terminology) [13]. It is associated to a resonant ring current in the entire SRR, which induces a magnetic dipole moment. The next three modes $\left(S_{1}, A_{2}\right.$, and $S_{2}$ ), have an alternate charge symmetry, as expected. In a plane perpendicular to the cross section of the $U$ path of the SRR, the charge distribution is almost symmetric (not shown) as usually observed in thin metallic nanoparticles [8] and nanoantennas (in that case the mode has an azimuthal number $m=0$ with full symmetry around its axis) in the quasistatic limit [21]. The presence of a substrate will
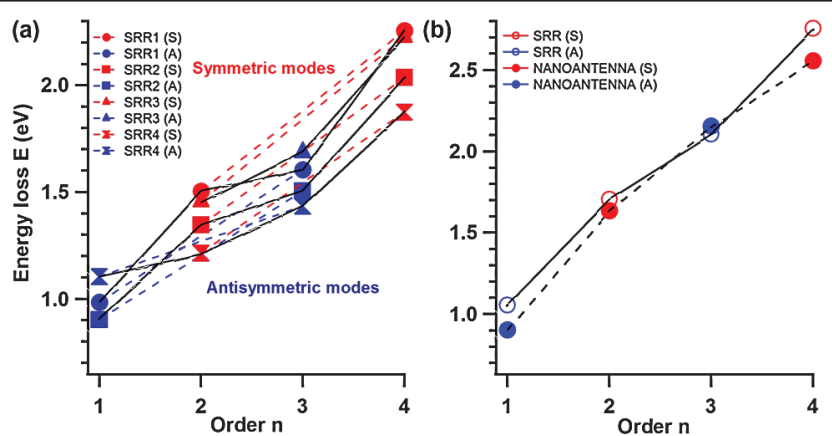

FIG. 3 (color). Dispersion curves for SRRs and a nanoantenna. (a) Experimental dispersion curves for slightly different sizes and shapes (these samples are labeled SRR1, SRR2, SRR3, and SRR4). Blue dashed lines: dispersion curves considering only the antisymmetric modes $(A)$. Red dashed lines: dispersion curves for only the symmetric modes $(S)$. The entire dispersion curves for all SRRs are plotted as black lines. We note the atypical behavior of the dispersion curve associated with antisymmetric modes of SRR4. Moreover, the magnetic mode of SRR3 could not be identified and therefore is not shown in the plot. (b) Dispersion curves from BEM calculations for a symmetric SRR (black line) and a straight nanoantenna (black dashed line).

only weakly modify this symmetry in the case of a small radius. At this point it should be stressed that the EELS gives consistent results when compared to optical measurements [22-24]. Moreover, EELS study allows us to detect all modes simultaneously on individual SRRs. Interestingly, the $S_{2}$ mode has never been observed in experiments before, to the best of our knowledge.

The EELS maps that we obtained for different SRRs clearly show that the spatial distribution of the resonances is not affected by small defects in the 3D shape of the SRR (not shown). This is a reassuring confirmation of the spatial coherence of surface plasmons when their wavelength is large compared to the defects, as it is the case here. Also, the measured maps are in good agreement with simulations, clearly indicating that, the surface plasmon distributions are very similar to those of standing waves in a nanoantenna, as already seen experimentally on two antisymmetric modes for much larger SRRs [20] and predicted by numerical simulations [10]. This situation hardly changes for asymmetric SRRs (not shown). Furthermore, the charge density eigenmodes (surface plasmon modes) are not influenced by the $U$ shape of the SRR, as compared to a nanoantenna [see Fig. 1(c), inset). It is thus surprising to note that the electric field distribution along a nanoantenna is symmetric in the plane perpendicular to its long axis (not shown) while this is not the case for the electric field distribution along the $U$ path of a SRR. Our calculations show indeed a concentration of vertical electric field lines on the outer part of the SRRs for some maxima [see Fig. 2(a)], due to the global shape of the SRR which is different from that of a nanoantenna. This explains why the EELS signal (sensitive to the $z$ EMLDOS) is concentrated 
on the outer part of the SRR for these maxima, although the charge density is almost the same as the equivalent $m=0$ mode of a nanoantenna. Our calculations also show that the contribution of the electric field along the electron path at the tips for both the SRR and nanoantenna is very weak for the modes $A_{2}$ and $S_{2}$ explaining the lack of EELS intensity observed at the tips for these modes.

The energy distribution of the modes displayed in Fig. 3(a) is also puzzling. For a nanoantenna, we expect that the different orders follow a concave dispersion law (linear in the case of very high aspect ratio), as predicted by numerical simulations $[25,26]$ and reproduced in Fig. 3(b). This is because they are essentially standing waves built onto the $m=0$ branch of an infinite cylinder. Now, the variation of the energy separation between the modes is not monotonic (especially the modes $S_{1}$ and $A_{2}$ are lying in close proximity), while the energy separation between symmetric modes on the one hand and antisymmetric modes on the other hand is almost the same. It is relevant to attribute this effect to the coupling between both legs of the SRR that will break the degeneracy of the initial dispersion relation into two barely parallel dispersion curves without affecting the charge density distribution to first order [see Fig. 1(c), inset]. In such case, the symmetric modes will stand in the high-energy branch, while the antisymmetric modes will lie in the lower branch, as experimentally observed.

We have studied small lithographed SRRs compatible with EELS study in which plasmonic resonances show up, so that the analysis of these split rings is not as straightforward as for larger rings that are well described by circuit theory. The basic behavior of the surface plasmon modes in SRRs is similar to the modes of nanoantennas of the same cross section while the spatial distribution of the electric field is different. However, the coupling between both legs of the SRR introduces a different ordering and energy spacing of the various modes, but the separation in symmetric and antisymmetric modes ( $S$ and $A$ ) of the nanoantenna can be still maintained in the rings. This work, describing the entire eigenstates of SRR, should give useful insight on the metamaterial they are built in. Moreover, the proposed experimental and simulations developments can be now extended to all $e$-beam-lithographed structures, opening the way to the full near-field characterization of a wide range of different metamaterials.

The authors acknowledge financial support from the European Union under the Framework 6 program under a contract for an Integrated Infrastructure Initiative. Reference 026019 ESTEEM.

*kociak@1ps.u-psud.fr

[1] V. Veselago, Phys. Usp. 10, 509 (1968).
[2] J. B. Pendry, Phys. Rev. Lett. 85, 3966 (2000).

[3] D. Schurig, J. Mock, B. Justice, S. Cummer, J. Pendry, A. Starr, and D. Smith, Science 314, 977 (2006).

[4] J. Zuo, M. Kim, M. O'Keeffe, and J. Spence, Nature (London) 401, 49 (1999).

[5] S. G. Lemay, J. W. Janssen, M. van den Hout, M. Mooij, M. J. Bronikowski, P. A. Willis, R.E. Smalley, L.P. Kouwenhoven, and C. Dekker, Nature (London) 412, 617 (2001).

[6] J. Pendry, A. Holden, D. Robbins, and W. Stewart, IEEE Trans. Microwave Theory Tech. 47, 2075 (1999).

[7] L. Novotny, Phys. Rev. Lett. 98, 266802 (2007).

[8] J. Nelayah, M. Kociak, O. Stéphan, N. Geuquet, L. Henrard, F. de Abajo, I. Pastoriza-Santos, L. LizMarzán, and C. Colliex, Nano Lett. 10, 902 (2010).

[9] S. Linden, C. Enkrich, M. Wegener, J. Zhou, T. Koschny, and C. Soukoulis, Science 306, 1351 (2004).

[10] C. Rockstuhl, F. Lederer, C. Etrich, T. Zentgraf, J. Kuhl, and H. Giessen, Opt. Express 14, 8827 (2006).

[11] B. Schaffer, U. Hohenester, A. Trügler, and F. Hofer, Phys. Rev. B 79, 041401(R) (2009).

[12] J. J. Cha, Z. Yu, E. Smith, M. Couillard, S. Fan, and D. A. Muller, Phys. Rev. B 81, 113102 (2010).

[13] C. Enkrich, M. Wegener, S. Linden, S. Burger, L. Zschiedrich, F. Schmidt, J.F. Zhou, T. Koschny, and C.M. Soukoulis, Phys. Rev. Lett. 95, 203901 (2005).

[14] J. Nelayah, M. Kociak, O. Stéphan, F. García de Abajo, M. Tencé, L. Henrard, D. Taverna, I. Pastoriza-Santos, L. LizMarzán, and C. Colliex, Nature Phys. 3, 348 (2007).

[15] A. Gloter, A. Douiri, M. Tencé, and C. Colliex, Ultramicroscopy 96, 385 (2003).

[16] F. J. García de Abajo and A. Howie, Phys. Rev. B 65, 115418 (2002).

[17] V. Myroshnychenko, E. Carbó-Argibay, I. PastorizaSantos, J. Pérez-Juste, L. Liz-Marzán, and F. de Abajo, Adv. Mater. 20, 4288 (2008).

[18] F. García de Abajo, Rev. Mod. Phys. 82, 209 (2010).

[19] F. J. García de Abajo and M. Kociak, Phys. Rev. Lett. 100, 106804 (2008).

[20] T. Zentgraf, J. Dorfmüller, C. Rockstuhl, C. Etrich, R. Vogelgesang, K. Kern, T. Pertsch, F. Lederer, and H. Giessen, Opt. Lett. 33, 848 (2008).

[21] L. Douillard, F. Charra, Z. Korczak, R. Bachelot, S. Kostcheev, G. Lerondel, P. Adam, and P. Royer, Nano Lett. 8, 935 (2008).

[22] M. Husnik, M. Klein, N. Feth, M. König, J. Niegemann, K. Busch, S. Linden, and M. Wegener, Nat. Photon. 2, 614 (2008).

[23] V. Shalaev, Nat. Photon. 1, 41 (2007).

[24] C. Soukoulis, S. Linden, and M. Wegener, Science 315, 47 (2007).

[25] R. Gómez-Medina, N. Yamamoto, M. Nakano, and F. Abajo, New J. Phys. 10, 105009 (2008).

[26] J. Dorfmüller, R. Vogelgesang, R. Weitz, C. Rockstuhl, C. Etrich, T. Pertsch, F. Lederer, and K. Kern, Nano Lett. 9, 2372 (2009). 